Histoire de l'histoire des sciences

\title{
ÉPISTÉMOLOGIE 1900 LA TRADITION FRANÇAISE
}

Enrico Castelli Gattinara

RÉsumé : L'article s'interroge sur la tradition française en matière d'épistémologie et analyse la réaction du milieu philosophique du début $d u x^{e}$ siècle face à la crise des acquis philosophiques traditionnels provoquée par le développement des sciences. Les interventions au premier congrès international de Philosophie de 1900 montrent que la philosophie essaie de continuer à exercer une hégémonie sur les sciences, mais que, pour réagir à la crise, elle doit s'engager dans l'histoire des sciences. L'histoire des sciences devient alors le moyen employé par la philosophie pour essayer de garder sa place privilégiée dans l'ensemble du savoir : elle est donc faite par des philosophes qui, de ce fait, en contrôlent les lieux institutionnels. Il devient dès lors possible de tracer une ligne qui relie les questions des philosophes de 1900 à celles qui préoccupent ceux de l'entre-deux-guerres, notamment Gaston Bachelard.

Mots-CLÉs : histoire des sciences, épistémologie, crise, premier congrès international de Philosophie.

ABSTRACT : The paper reviews the French tradition on epistemology and the reaction of the philosophical milieu in the early $\mathrm{xx}^{\text {th }}$ century against the crisis of philosophical concepts induced by the development of science. The $1^{\text {st }}$ International Congress on Philosophy of 1900 in Paris shows that philosophy tries to confirm her hegemony on sciences, but for this, philosophers have to embark into history of science. History of science becomes for philosophy the means to keep the highest place in the whole realm of knowledge: philosophers become historians of science and supervise historical institutes. It is still possible to show quite a continuity between the philosophical questions of 1900 and some similar problems raised in the years between the two world wars, especially by Gaston Bachelard.

KEYWORDS : history of science, epistemology, crisis, $1^{\text {st }}$ International Congress on Philosophy.

Revue de synthèse : $4^{\mathrm{e}}$ sér., ${ }^{\text {os }}$ 2-3-4, avr.-déc. 2001, p. 347-365. 
Zusammenfassung : Der Artikel behandelt die französische Tradition der Epistemologie und analysiert die Reaktion der Philosophen des frühen 20. Jahrhunderts auf die Krise der philosophischen Konzepte, die von der Entwicklung der Naturwissenschaften ausgingen. Der Erste Internationale Kongreß für Philosophie, der im Jahre 1900 in Paris stattfand, zeigte, daß die Philosophie damals versuchte, ihre Herrschaft über die anderen Wissenschaften zu behalten. Dazu benötigte sie die Wissenschaftsgeschichte, um ihre eigene Krise zu bewältigen und um ihren obersten Platz in der Hierarchie des Wissens zu behalten. Folglich wurde Wissenschaftsgeschichte oft zum Arbeitsgebiet von Philosophen, die dadurch auch deren Institutionen kontrollierten. Seitdem läßt sich eine Kontinuität nachweisen, die von den philosophischen Problemen der Jahre um 1900 zu denjenigen Fragen führt, die in der Zeit zwischen den Weltkriegen insbesondere von Gaston Bachelard aufgeworfen werden sollten.

STICHWÖRTER: Wissenschaftsgeschichte, Epistemologie, Krise, Erster internationaler Kongress für Philosophie.

Enrico Castelli Gattinara, né en 1959, professeur et directeur de la revue Aperture, s'intéresse à l'épistémologie et à la théorie de l'histoire. Outre plusieurs articles, il a publié Epistemologia e storia (Milan, Franco Angeli, 1996) et Les Inquiétudes de la raison (Paris, Vrin/ EHESS, 1998). Il enseigne l'épistémologie de l'histoire à l'université Rome 1 (Italie).

Adresse : 11, via A. Allegri da Correggio, I-00196 Rome.

Courrier électronique : aperture@tiscalinet.it 
NOUVEAU SIÈCLE, NOUVEAUX PROBLÈMES

Y a-t-il en France une tradition épistémologique? La réponse ne peut naturellement qu'être affirmative si l'on considère le rôle que l'histoire des sciences y a joué. Il est intéressant de noter que cette tradition s'impose en France au début $\mathrm{du} \mathrm{xx}^{\mathrm{e}}$ siècle, à un moment où les certitudes philosophiques ne reposent plus sur un fondement solide et où les sciences, par leurs découvertes et notamment par la formulation de nouvelles théories, ne semblent guère respecter les points fixes de la raison philosophique.

Début 1900 : tout le monde connaît cette période comme une période de « crise ». J'ai insisté, ailleurs ${ }^{1}$, sur le fait qu'il ne fallait pas parler de cette crise au singulier, dans la mesure où il y eut en réalité une multiplicité de crises différentes qui ont affecté différentes régions du savoir et se sont étalées sur une période assez longue, au moins quelques décennies. Néanmoins, les gens de l'époque, philosophes, artistes, savants, hommes politiques, etc., ont bien perçu leur époque comme une époque de crise. C'est au tournant du siècle que l'on assiste à une augmentation sensible des articles et des livres consacrés à des problèmes méthodologiques au sein des sciences, une conséquence directe de l'inquiétude des philosophes. La question débattue ici sera la suivante : s'il est vrai qu'il y a une tradition épistémologique française, quel a été son enjeu principal au moment de sa naissance? Autrement dit, qu'est-ce qui la constitue comme tradition malgré ses différences internes? Il suffit de lire les textes des philosophes épistémologues français des premières décennies $\mathrm{du} \mathrm{xx}^{\mathrm{e}}$ siècle pour s'apercevoir que le rapport philosophie/sciences, qui avait toujours été l'un des axes privilégiés de la pensée française, est à son tour entré en crise. Mais il y a une spécificité toute française dans la manière de réagir à cette crise qui devait contribuer à définir la philosophie des sciences et l'épistémologie hexagonales. Cette spécificité correspond à l'idée que, face à la crise indéniable qui touche la Raison, on n'a pas le droit de renoncer à son pouvoir et d'en déclarer la banqueroute. On peut par contre sauvegarder la raison scientifique en considérant les aspects positifs de la crise et en s'ouvrant aux acquis de l'histoire, qui nient toute valeur aux discours sur la «faillite de la science» qui circulent depuis les toutes dernières années $1800^{2}$.

1. Castelli Gattinara, 1997.

2. Voir le célèbre texte de Brunetière, 1895. 
Le rôle de la philosophie elle-même est alors en jeu dans la mesure où, face aux bouleversements provoqués par les nouvelles théories scientifiques qui s'enchaînent dans plusieurs domaines (mathématiques, physique, chimie, biologie), elle risque de perdre sa fonction de «guide » pour la pensée et pour le savoir en général; et ce, d'autant plus que des savants de l'époque, comme Henri Poincaré et Pierre Duhem, étaient dotés d'un esprit philosophique qui était clairement apparu dans des textes devenus immédiatement fondamentaux.

Les philosophes ne peuvent donc plus assister passivement à la leçon des sciences. 1900 représente en France le siècle nouveau, le siècle inauguré par la grande Exposition universelle et par le premier congrès international de Philosophie, le siècle où la philosophie - conformément aux souhaits des philosophes de la génération précédente, notamment Émile Boutroux et Félix Ravaisson, mais aussi déjà, bien avant eux, Auguste Comte et Antoine Augustin Cournot, qui appelaient les philosophes à s'occuper des sciences - s'intéresse aux sciences tout en essayant d'en ordonner sinon le cours, du moins la structure rationnelle et la logique ${ }^{3}$. Ce qui est en jeu, semble-t-il, ce n'est rien de moins que la possibilité de tenir un discours sur la science, au-delà des pratiques scientifiques spécifiques et des stratégies de la recherche. Des problèmes nouveaux se posent. Les vieilles querelles philosophiques sont alors reformulées et revues en fonction des nouvelles découvertes scientifiques. Des querelles nouvelles s'imposeront, qui ne reprendront qu'en partie les vieux problèmes philosophiques. On le perçoit aisément à travers les débats qui ont émaillé la première moitié $\mathrm{du} \mathrm{xx}^{\mathrm{e}}$ siècle, notamment les deux polémiques les plus importantes du début du siècle, qui ont en un certain sens inauguré la série : celle entre Bertrand Russell via Louis Couturat et Henri Poincaré (logicisme versus réalisme mathématique) et celle, en apparence plus «française», entre Pierre Duhem et Abel Rey (énergétisme versus mécanisme).

La question générale qui anime ces polémiques est toujours celle du cadre conceptuel et théorique permettant d'interpréter l'ensemble des connaissances scientifiques : une très vieille querelle philosophique - en dernière instance, le choix entre réalisme et nominalisme - s'articule alors d'une façon nouvelle, en engageant dans le débat non seulement des philosophes, mais aussi des mathématiciens, des physiciens et des logiciens de différents pays. Poincaré et Rey incarnent dans ce cas une tradition proprement française, qui défend les droits d'un réalisme laïc, objectiviste et

3. Voir, p. ex., Rey, 1907, p. 498 : « Tout en reconnaissant que les questions générales (de méthode, d'esprit, d'idées directrices), en matière des sciences, sont encore du ressort des savants, que nul mieux que les praticiens professionnels n'est qualifié pour en aborder la philosophie, il faut reconnaître que la pratique de la science ne suffit pas à résoudre tous les problèmes logiques et philosophiques qu'elle peut poser. » 
rationnel, contre la tendance du formalisme à enserrer le réel en le vidant de ses aspects «intéressants et importants ${ }^{4}$ ». C'est à peu près dans les mêmes années que naît officiellement en France l'épistémologie comme branche de la philosophie, si l'on considère l'œuvre d'Émile Meyerson, Identité et réalité ${ }^{5}$, comme la première où le terme d'épistémologie est employé au sens où on l'entend aujourd'hui. Cette coïncidence n'est pas due au hasard, car elle répond à une exigence de réflexion sur les fondements de la connaissance scientifique qui, à l'époque, devient de plus en plus nécessaire aux yeux des philosophes.

UN CONGRÈS POUR INDIQUER LA TÂCHE UNITAIRE DE LA PHILOSOPHIE

Ce besoin de réflexion s'exprime pour la première fois de façon explicite lors du premier congrès international de Philosophie qui a lieu à Paris au début du mois d'août 1900. Le mot «épistémologie », en effet, est employé par Jean Wilbois ${ }^{6}$ (et ensuite par Russell) au lieu de la formule « critique des sciences » ou « critique générale des sciences » utilisée à l'époque pour indiquer la réflexion philosophique sur les sciences - on pourrait ainsi faire remonter la date de naissance officielle de l'épistémologie à 1900, si la question avait quelque intérêt. Or, ce congrès international représente en quelque sorte l'acte de naissance d'un souci spécifiquement épistémologique, car son caractère international est dû à la nécessité de rassembler toutes les forces disponibles pour faire face aux pouvoirs croissants de ce que nous appelons aujourd'hui les technosciences.

Pour les philosophes, il ne s'agit pas de faire face aux sciences théoriques, à la science en général ou aux théories abstraites : l'objet de leur principale préoccupation est bel et bien ce qu'ils appellent «les sciences et les techniques » dans leur efficacité et dans leurs applications pratiques.

«D'une part, la philosophie s'est rapprochée des sciences en les voyant atteindre et résoudre les problèmes réels et vivants dont elle ne peut se désintéresser : et réciproquement les savants ont éprouvé le besoin, après l'enthou-

4. Il y avait, en France, une forte tradition non-laïque qui s'était rangée du côté du nominalisme : Pierre Duhem en était le meilleur exemple et s'inspirait de l'énergétisme de Wilhelm Ostwald ; mais on peut également citer Édouard Le Roy, qui était pour ainsi dire un «nominaliste pur ». Tous les deux étaient catholiques, mais il ne faut pas oublier que parmi les catholiques français, il y avait aussi un fort courant antiscientiste dont faisaient partie les théoriciens de la «faillite de la science ».

5. Meyerson, 1908.

6. Wilbois, 1900 , p. 503-698, en part. p. 540. 
siasme que la science pour la science avait d'abord provoqué, de compléter leur œuvre et de lui donner un sens général par une synthèse philosophique des conclusions fragmentaires qu'ils avaient déjà établies. La philosophie, dans cette association, n'a pas perdu son caractère pour devenir un simple prolongement de chaque science positive. Elle le dirige autant qu'elle les continue ${ }^{7}$. »

Le congrès est en effet lié à l'Exposition universelle de 1900. Les Expositions universelles, qui se tenaient depuis 1855 , n'avaient pas manqué de susciter l'appréhension des philosophes, comme le démontre l'intervention inaugurale d'Émile Boutroux qui rappelle le discours fait en 1855 par Ernest Renan, où celui-ci exprimait sa crainte que « ces pompeuses manifestations de l'industrie et des sciences mécaniques ne fussent, en définitive, que des simples fêtes de la matière ». Rattachant le congrès des philosophes à l'Exposition universelle de 1900, on donne alors la preuve, comme le dit Boutroux, que dans « nos sociétés en apparence absorbées par l'exploitation de la matière », les idées et les valeurs humaines occupent encore une place importante. «L'Exposition [...] représente essentiellement l'alliance de l'industrie et de la science, du travail et de la pensée [...], du réel et de l'idéal », le congrès est donc la « fête de l'esprit ennoblissant la matière $^{8} \gg$. Il ne faut plus que la philosophie soit méfiante à l'égard des sciences, mais il ne faut pas non plus que les sciences absorbent la philosophie ou qu'elles éliminent toute espèce d'élément philosophique. L'alliance science-philosophie, dont le congrès doit être le point de départ, ne peut plus avoir l'aspect d'un assujettissement de l'une à l'autre.

Les philosophes constatent d'ailleurs bien que leur terrain diminue de plus en plus et que la connaissance du réel ne leur appartient plus. La philosophie semble vraiment une sorte «d'empire au moment de sa décadence ». La constitution des sciences humaines la dépossède de ses objets même dans ses domaines «classiques » (la psychologie, la réflexion sur la société, la politique, l'anthropologie, etc., ces «branches de la philosophie », comme le disait Boutroux); ils ne sont plus exclusivement cernés par une spéculation abstraite, mais rapprochés de considérations d'ordre expérimental. Les sciences sont en effet de plus en plus capables d'amener « dans l'étude du réel, de la vie, de l'âme même, la rigueur et la certitude que [la philosophie] n'atteignait jadis que dans l'ordre des abstractions et des possibilités ${ }^{9} »$. Que reste-t-il donc ? Ce sur quoi la science n'a aucune prise : la morale, la métaphysique et l'histoire de la philosophie.

7. Boutroux, 1900. La citation est reprise de l'intervention présentée oralement au Congrès, qui ne correspond pas exactement à celle publiée dans la Revue de métaphysique et de morale, voir LALANDE, 1900, p. 483.

8. Boutroux, 1900, p. 503-504.

9. Boutroux, 1900, p. 505. 
Évidemment, il faut «maintenir l'originalité et l'autonomie relative de la philosophie », et même « lui assigner une tâche qui, née de la réflexion originale de l'esprit sur les connaissances scientifiques, dépasse véritablement la portée et les méthodes des sciences particulières ${ }^{10}$. Car la raison humaine ne se réduit pas à l'accumulation des connaissances scientifiques : son but est celui de l'unification et de la synthèse. C'est pourquoi la philosophie cherche à assimiler tous les domaines de la connaissance expérimentale qui sont propres à l'enrichir et à la fortifier. Dans cette perspective, la philosophie maintient le privilège d'une discipline qui la met directement en rapport avec les sciences, et qui est à l'époque en train de se formaliser et de se spécialiser énormément : la logique. Celle-ci est véritablement d'ordre métascientifique, et elle peut représenter la métaphysique dans l'ordre rigoureux de la raison scientifique. D'où l'attention qu'elle obtient au congrès de 1900 : une section est intitulée «logique et histoire des sciences ».

De plus, c'est le travail même du philosophe qui doit changer, car il n'est plus question de le considérer comme le travail solitaire d'un esprit de génie qui ferait la synthèse de toutes les connaissances : «Ce qu'un individu ne peut embrasser, les hommes de tous les pays, réunis pour échanger leurs connaissances et se compléter les uns les autres [...] doivent peu à peu le rassembler » et obéir de cette façon à ce qui reste «le postulat» de la philosophie, à savoir l'unification ${ }^{11}$. Voilà pourquoi il faut se mettre «à l'école des sciences de la nature, sans s'y enfermer toutefois ». Geste d'humilité apparente afin de permettre l'exercice d'un pouvoir d'unification et de mise en ordre qui maintient le philosophe dans une dimension supérieure, encore une fois métaphysique.

Si la philosophie a un objectif, c'est bien celui de reconnaître une « harmonie » au sein du tout et de chercher une raison dans les choses, car cette raison semble échapper à toute prise alors que les choses sont considérées simplement comme marchandises. Chercher une raison harmonique dans les choses veut donc dire rétablir un ordre de la raison au sein d'un monde matériel et industriel que les «technosciences» rendent de plus en plus autonome par rapport aux valeurs et aux idéaux.

Le souci des philosophes est donc disciplinaire au sens strict du terme. Les premières conférences données au congrès lors de la première journée portent, en effet, sur l'enseignement de la philosophie, son état, ses conditions et ses tâches, car il faut avant tout discipliner les élèves pour les rendre sensibles aux idées abstraites, remarque François Evellin, alors inspecteur d'académie. Mais l'intention disciplinaire apparaît dans toute sa

10. Boutroux, 1900, p. 505.

11. Boutroux, 1900, p. 509. 
splendeur dans la conférence du R. P. Bulliot, professeur à l'Institut catholique de Paris, qui laisse aussi apparaître la crise d'identité du philosophe : «Avant d'unifier la science, la philosophie devra donc s'unifier ellemême »; mais pour atteindre ce but, elle devra «se mettre résolument à l'école de la science », puisque c'est seulement ainsi qu'elle pourra trouver les données qui lui sont nécessaires. Bulliot tient à spécifier que « la philosophie n'a nullement à craindre, en suivant cette voie, de se voir absorbée par la science, car la science et la philosophie se distingueront toujours l'une de l'autre par leur finalité et par leurs lumières propres ». La science s'intéresse en effet aux phénomènes et aux causes immédiates, alors que la philosophie «embrasse l'ensemble des choses» et «peut donner à une classe de phénomènes [...] son interprétation définitive ».

L'ordre de la raison que la philosophie doit établir, l'interprétation définitive d'une classe quelconque de phénomènes faite au nom d'une unification qui devrait dépasser les limites des sciences, n'est toutefois plus un acquis, car autrement Bulliot n'aurait pas ressenti la nécessité de souligner à plusieurs reprises que la philosophie ne risque pas d'être absorbée par des disciplines qui semblent vouloir en prendre la place, ou du moins rendre sa place inutile, voire contradictoire. Et voilà la conclusion magistrale de Bulliot, qui confirme l'enjeu du congrès : «Bien loin donc de se laisser absorber par la science, elle contrôle ses hypothèses et ses théories; elle informe de son esprit les intelligences scientifiques; elle reste [...] la science par excellence, architectonique et maîtresse ${ }^{12}$.»

On retrouve aussi la même tonalité disciplinaire dans les conférences des autres sections, notamment celles consacrées à l'histoire de la philosophie et à la morale. Elle est toutefois absente de la section consacrée à la logique et à l'histoire des sciences, qui rassemble non seulement des philosophes mais aussi (et surtout) des savants. Dans cette section, les discussions sont en effet moins concordantes, et les diverses interprétations des théories et des connaissances scientifiques entrent souvent en conflit. On y voit dialoguer, par exemple, Henri Poincaré avec Bertrand Russell, Louis Couturat ou Paul Painlevé, ou encore Moritz Cantor et Paul Tannery, Jacques Hadamard et Édouard Le Roy.

Il n'en reste pas moins que le souci de l'unité se manifeste là aussi comme l'un des soucis principaux. Toutefois, comme le remarque Poincaré, dont plusieurs conférenciers avaient critiqué le «scepticisme », il ne faut pas discuter dans cette section les questions proprement philosophiques qui doivent être traitées dans la section de métaphysique. Tout se passe donc comme s'il devait y avoir une division du travail intellectuel ou spéculatif, afin de séparer les questions d'ordre épistémologique (ou

12. Boutroux, 1900, p. 523-524 (souligné par moi). 
méthodologique, par exemple le déterminisme) des questions d'ordre philosophique (ou métaphysique, par exemple la réalité du monde extérieur). Mais nous savons que Poincaré a été plus savant que philosophe et qu'il est conscient, plus que tout autre, qu'il n'y a rien à craindre du côté des sciences.

Il y a donc, selon les interventions du congrès, une différence d'enjeu entre les conférences des sections «philosophiques » et celles de la section «épistémologique » (remarquons, de surcroît, que le poids de la « logique » dépasse largement en quantité celui de l'histoire des sciences); une différence due surtout à la présence de savants dans cette section, qui sont moins «en crise » que les philosophes devant l'évolution des sciences et de leurs implications conceptuelles (à savoir la mise en question épistémologique des certitudes concernant le déterminisme, l'approximation des connaissances, le conventionnalisme méthodologique, le rôle des hypothèses, etc.).

Dans les années qui suivent, et même dans les décennies suivantes, les philosophes maintiennent cette attitude de «chercheurs d'ordre », et leurs arguments restent des arguments d'autorité. Les problèmes soulevés par le premier congrès international de Philosophie sont repris dans les congrès suivants, et les questions épistémologiques sont traités avec un intérêt toujours croissant. Par rapport aux premiers congrès, un seul changement a lieu au fil des décennies : l'histoire des sciences, décidément minoritaire dans l'ensemble des questionnements philosophiques sur les sciences du tout premier congrès, prend plus de place et parvient, mais seulement à partir de 1929, à organiser des congrès internationaux qui lui sont exclusivement consacrés. Entre-temps, l'histoire des sciences ne demeure évidemment pas inactive et s'organise sous l'aile «protectrice» (en réalité hégémonique) de l'histoire générale : c'est le cas, par exemple, du congrès d'Histoire des sciences qui a lieu lui aussi en 1900, juste avant le premier congrès international de Philosophie, et qui est rattaché au congrès international d'Histoire comparée comme cinquième section.

Il ne faut toutefois pas sous-estimer le choix du congrès des philosophes d'associer la logique (en plus de la logique en sens propre, ce mot désigne aussi la logique de la connaissance scientifique, l'épistémologie) et l'histoire des sciences. Ni se méprendre sur le dédoublement, assez unique en son genre, d'une discipline représentée à la fois dans un congrès d'histoire 
et dans un congrès de philosophie ${ }^{13}$. Les philosophes savaient, en effet, depuis longtemps qu'il fallait s'occuper aussi d'histoire des sciences, comme l'avait écrit Boutroux, s'ils voulaient non pas suivre passivement le cours évolutif des sciences, mais lui donner un ordre et une unité. L'histoire des sciences n'a toutefois pas de statut déterminé, ni de place dans le monde académique (Tannery, qui est l'un de ses représentants les plus réputés, ne fut pas nommé à la chaire d'histoire des sciences au Collège de France ${ }^{14}$ ). Malgré la bonne volonté de ceux qui s'y intéressent, qui ne sont pas des spécialistes au sens d'une «professionnalisation » stable en histoire des sciences, mais surtout des savants ou des philosophes (Duhem et Tannery sont ici des cas emblématiques), elle était tiraillée aussi bien du côté de l'histoire que du côté de la philosophie.

Cela n'a d'ailleurs rien d'étonnant, si l'on songe au fait que le président de la $V^{\mathrm{e}}$ section «Histoire des sciences » du congrès d'Histoire comparée de 1900 est précisément Paul Tannery, et que l'un des deux vice-présidents est le philosophe André Lalande (tous les deux présents, la semaine suivante, au congrès de Philosophie, dans la section «Logique et histoire des sciences $\gg$ ).

En outre, parmi les sections consacrées aux grands thèmes philosophiques du congrès de 1900 , seule existe une section vouée à l'histoire des sciences. Certes, celle-ci apparaît comme un champ spécifique assez restreint si on la compare à l'importante section réservée exclusivement à l'histoire de la philosophie - bien que cette importance soit bien légitime dans un congrès de philosophie. Mais ni l'histoire de l'art, ni l'histoire littéraire, ni l'histoire politique ne sont prises en considération. Les sciences jouissent donc d'un statut très favorable. Surtout en France - malgré ce que répètent souvent les philosophes et que Boutroux lui-même rappelle dans son allocution initiale en laissant entendre que les philosophes ne s'occupent pas suffisamment des sciences - où les sciences occupent depuis toujours une place importante, ainsi que leur histoire puisqu'elle coïncide souvent avec l'histoire de la philosophie.

Dans son intervention finale au congrès de Philosophie, à la suite des remarques de plusieurs conférenciers qui avaient souligné l'importance de la section de «logique et histoire des sciences » du point de vue de la quantité des intervenants et de la participation de l'auditoire, Tannery propose de scinder celle-ci en deux sections distinctes : «[...] une section consacrée à la théorie de la connaissance scientifique; une section consacrée à l'his-

13. Je peux donc compléter ce que j'ai analysé dans mes travaux de ces dernières années, dont Ernest Coumet a bien voulu suivre l'évolution, car c'est bien avant les années vingt que l'histoire des sciences commence à jouer un rôle stratégique et intermédiaire entre l'histoire et la philosophie, dans le cadre d'une réflexion épistémologique qui était alors tout juste en train de se faire.

14. Voir, à ce propos, Coumet, 1981. 
toire des sciences ", et il y ajoute le vœu d'une fusion entre cette section et la section correspondante au sein du congrès d'Histoire comparée ${ }^{15}$. Le souci principal de Tannery est en effet de donner toute son importance à l'histoire des sciences, dans la mesure où elle seule pouvait montrer le caractère non-absolu des théories de la connaissance. Mais les philosophes sont alors attentifs à ne pas laisser échapper cette histoire, qui se révélera par la suite une alliée précieuse.

De fait, ce vœu était alors encore loin de se réaliser, car si les historiens généralistes, à l'époque, ne considèrent l'histoire des sciences que comme une spécialisation (d'ailleurs assez difficile, vu son objet), les philosophes ont tout intérêt à la faire jouer au profit d'une théorie de la connaissance. Les œuvres d'Émile Meyerson en témoignent à partir d'Identité et réalité (1908); et celles de Léon Brunschvicg le font également à partir de Les Étapes de la pensée mathématique (1912). La question à laquelle l'histoire doit répondre concerne l'ordre de la nature : la nature est-elle ordonnée en elle-même, et la tâche de la connaissance scientifique n'est-elle que la découverte de cet ordre caché, ou bien la réalité est-elle ordonnée par notre pensée? Une question d'ordre qui devient aussi une question cruciale, car le rôle de la philosophie doit rester, selon le désir des philosophes, la conservation de l'unité rationnelle du savoir sous toutes ses formes - unité qui doit élever l'esprit de l'humanité et le pousser à abandonner des intérêts purement matériels. Mais l'unification des sciences doit se faire en connaissance de cause, et l'histoire des sciences peut y contribuer.

La question de l'unité de la connaissance, et donc de l'unité des sciences, est appelée à l'époque une question de «morale ». Contrôler les diverses connaissances scientifiques pour en indiquer l'unité fondamentale est la tâche «morale » du philosophe : celui-ci doit en effet comprendre la «valeur » de la connaissance rationnelle au-delà de ses formes extérieures, sans pour autant les nier ou les ignorer ${ }^{16}$. En lisant les actes du premier congrès international de Philosophie, ce double souci concernant la théorie de la connaissance scientifique et la morale saute aux yeux : il est le signe éminent d'une crise qui s'exprime par la prépondérance d'une section sur les autres ${ }^{17}$.

D'où, évidemment, l'argument d'autorité que philosophes et scientifiques énoncent du haut de leur prestige : le sommet de la pensée humaine est représenté par la connaissance rationnelle, et plus spécialement par la

15. TANneRY, 1900, p. 695.

16. De telles positions étaient très fréquentes, même en dehors du milieu scientifique et philosophique. Elles étaient aussi soutenues par un littéraire influent comme Paul Valéry qui, tout au long de sa vie, maintint une attention très vive pour les questions scientifiques et épistémologiques; voir VALÉRY, 1973.

17. Boutroux, 1900, p. 505-506. 
connaissance scientifique. La tâche de la philosophie sera donc désormais de démontrer cette priorité que les sciences, absorbées par leurs recherches, ne peuvent formuler. D'autant plus qu'en progressant et en se spécialisant, la connaissance scientifique devient toujours plus problématique, et qu'au lieu de résoudre les problèmes, elle en pose de nouveaux. Une belle définition de Paul Valéry peut nous servir à ce propos :

«Une science réelle n'est pas un système de réponses. Au contraire, c'est un système de problèmes qui demeurent toujours ouverts. Les axiomes fondamentaux d'une science sont les déterminations partielles des problèmes ${ }^{18}$. »

De ce premier argument d'autorité découlait cependant un second : toute connaissance scientifique est, en tant que telle, une connaissance rationnelle. Il faut donc que la philosophie, selon une tradition que l'on fait remonter à Kant, puisse faire reconnaître la connaissance scientifique comme une connaissance rationnelle, ce qui ne va pas de soi à l'époque (il faut en effet définir ce qui est rationnel et ce qui ne l'est pas, d'où l'une des disputes les plus vives du début du siècle entre énergétistes et mécanistes). Les sciences semblent d'ailleurs ne pas tenir compte du travail du concept et des efforts de la philosophie, et partent dans toutes les directions en abordant des questions nouvelles sur lesquelles les philosophes ont du mal à conceptualiser et à catégoriser : il en est ainsi des mathématiques, de la physique, de la biologie et, vers la fin du $\mathrm{XIX}^{\mathrm{e}}$ siècle, des très jeunes sciences humaines (pour ne mentionner qu'un exemple très connu, la naissance de la psychanalyse freudienne et ses dérives jungiennes ${ }^{19}$ ).

L'attention portée à l'histoire des sciences doit donc servir la théorie de la connaissance, c'est-à-dire l'épistémologie, pour suivre, sinon pour contrôler, le développement des recherches scientifiques et aider les philosophes à établir des points de repère à partir desquels le travail des savants puisse être jugé. C'est en France que se manifeste le plus fortement ce souci, bien plus que dans d'autres pays ${ }^{20}$. Les philosophes français semblent, en effet, davantage concernés et bien que, pendant le congrès philosophique de 1900, les savants et les philosophes d'autres pays aient été moins exigeants à l'égard d'une théorie unifiée de la connaissance rationnelle (par exemple, l'italien Alessandro Padoa), l'alliance science-

18. VALÉRY, 1973, vol. II, p. 833 (il s'agit d'une note de 1901).

19. Le rapport complexe de Sigmund Freud à la rationalité scientifique est un problème très important : il ne peut être épuisé ici en quelques lignes.

20. Cela ne veut pas dire qu'à l'étranger il n'y avait pas d'approches semblables à la question : en Allemagne, p. ex., le courant néokantien de l'école de Marburg et, en part., la pensée d'Ernst Cassirer avaient plusieurs points en commun avec l'utilisation stratégique de l'histoire des sciences et sa valorisation philosophique, mais en France cela a donné lieu à ce que j'appelle une «tradition épistémologique » dont Alexandre Koyré, Gaston Bachelard et Georges Canguilhem sont les derniers représentants. 
philosophie-histoire des sciences souhaitée par Tannery se réalise surtout grâce aux Français, au nom de ce que Boutroux avait appelé alors « le postulat de l'unité ».

\section{LES ENJEUX « HUMANISTES » D'UNE HISTOIRE PHILOSOPHIQUE DES SCIENCES}

Le courant qui devient dominant en France cherche à continuer à maintenir ensemble, d'une façon qui, au fil des années, devient de plus en plus évidente, la réflexion épistémologique et l'histoire des sciences. Toute l'histoire des sciences, ou presque, que l'on fait en France dans les premières décennies $\mathrm{du} \mathrm{xx}^{\mathrm{e}}$ siècle, notamment à partir des années vingt, est faite sous l'aile protectrice (et directrice) de la philosophie. C'est d'ailleurs ce qui a été annoncé au congrès philosophique de 1900, lorsque Henri Berr y a présenté la Revue de synthèse historique qu'il venait de fonder et dont l'enjeu était précisément de rapprocher la réflexion théorique et la connaissance historique, avec une attention particulière consacrée aux sciences.

Il est aujourd'hui possible de regarder cette tradition comme une ligne de force tracée à partir de 1910 jusqu'aux années soixante. Il est en outre remarquable qu'à partir d'une certaine époque, notamment entre 1929 et 1934, soient créés une série d'instituts et de revues dont le but est spécifiquement l'histoire des sciences ${ }^{21}$, mais qui comprennent un nombre assez important de philosophes (qui en sont en général les fondateurs et suivent parfois des parcours théoriques qui s'opposent). On pourrait dire qu'une certaine tradition française d'histoire des sciences y mûrit, celle notamment qui se réfère à Hélène Metzger et à Alexandre Koyré bien plus qu'à Gaston Bachelard. Cette tradition, qui n'est évidemment pas homogène et se nourrit de conflits théoriques importants sur ce qu'est la science et la manière dont il faut en faire l'histoire, naît donc à partir d'une amitié particulière, d'une solidarité (le terme latin sodalitas, sodalicium serait plus approprié) entre l'histoire des sciences et la philosophie, une amitié que l'on trouve difficilement à l'étranger. Cette sodalitas se constitue comme une véritable tradition, selon un axe qui traverse l'œuvre d'auteurs à bien des égards assez différents, et se prolonge pendant presque soixante ans : je me réfère à la lignée qui va de Léon Brunschvicg et d'Abel Rey à Alexandre Koyré

21. Dans la mesure où j'en ai déjà parlé dans des articles publiés dans cette revue, voir Castelli Gattinara, 1998a, il suffira ici de rappeler qu'il s'agit de la revue fondée par Abel Rey, Thalès, de son Institut d'histoire et de philosophie des sciences, des sections qui y sont consacrées au Centre international de synthèse de Henri Berr et de la revue Archeion dirigée par Aldo Mieli. Beaucoup de philosophes ont d'ailleurs participé au premier congrès international d'Histoire des sciences de 1929. 
et qui aboutit à Georges Canguilhem. Quelques titres d'ouvrages et quelques noms d'institutions suffisent à le montrer: Brunschvicg écrit Les Étapes de la pensée mathématique (1912); Rey fonde l'Institut d'histoire et de philosophie des sciences (1932); Metzger publie l'essai La Méthode philosophique dans l'histoire des sciences (1937); Koyré publie les Études d'histoire de la pensée scientifique (1960); Canguilhem publie les Études d'histoire et de philosophie des sciences (1968).

Nous connaissons cette tradition par l'histoire de la "pensée » scientifique de Koyré et l'histoire épistémologique des sciences chère à Bachelard: deux philosophes auxquels l'histoire des sciences en France doit beaucoup. Mais, il faut le souligner, il s'agit bien de philosophes, en particulier dans le cas de Bachelard. Et ces philosophes ont une idée très précise de l'histoire des sciences; mieux : ils ont une idée précise de l'usage de cette histoire. Bachelard est emblématique de ce point de vue, puisqu'il écrit en 1938, dans La Formation de l'esprit scientifique, que l'histoire qui intéresse l'épistémologue doit être une histoire "purifiée », "sanctionnée », nettoyée de tous les détails et de toutes les incrustations, de tous les héritages et de tous les complexes qui la rattachent à son temps ${ }^{22}$.

Aucun philosophe n'a été aussi loin, mais Bachelard, dont le caractère personnel assez peu conformiste est connu, pouvait se permettre de telles provocations. Se présentant comme «philosophe historien», il explique que :

«[...] l'histoire des sciences ne peut être tout à fait une histoire comme les autres. Du fait même que la science évolue dans le sens d'un progrès manifeste, l'histoire des sciences est nécessairement la détermination des successives valeurs de progrès de la pensée scientifique ${ }^{23}$.»

Cependant, il faut attirer l'attention sur le fait que l'argument d'autorité que la philosophie cherche à imposer à l'histoire des sciences et aux sciences ne date pas des années trente mais remonte au début du siècle.

La difficulté de la philosophie, qui pousse des épistémologues comme Bachelard à refuser nettement la tradition cartésienne, se manifeste avant même les révolutions physiques les plus connues (la relativité et la mécanique quantique). Il s'agit d'une difficulté relative, certes, qui oblige toutefois en 1900 à donner plus d'importance à la théorie de la connaissance scientifique qu'à la morale, et qui, de plus, rend nécessaire la recherche d'un nouvel équilibre pour rendre à la morale sa juste place dans le règne de la métaphysique. Cependant, cette difficulté ne se traduit pas exclusivement par un refus net et presque sans appel de la raison scientifique

22. BACHELARD, 1938 , p. 250-251.

23. BACHELARD, 1972, p. 138 (souligné par moi). 
(comme ce sera le cas par exemple en Allemagne, chez Martin Heidegger $^{24}$ ), ou par le partage proposé par Henri Bergson entre la pensée scientifique quantitative et la pensée métaphysique qualitative; elle se manifeste en revanche en France par la ferme tentative de sauvegarder la valeur de la rationalité scientifique humaine. Le travail de Bachelard s'insère d'ailleurs dans cet effort, et en est en quelque sorte l'un des produits. Bachelard indique, effectivement, plus explicitement que tous les autres, le rôle que la philosophie doit avoir à l'égard de la connaissance scientifique, sans complexe d'infériorité et sans incompétences : «Nous croyons travailler ainsi à la moralisation de la science ${ }^{25}$. 》

Face à des sciences qui se développent toujours davantage et semblent indifférentes aux conséquences conceptuelles de leurs acquis, la philosophie des sciences, en se spécialisant en épistémologie, répond en se donnant un rôle «moralisateur » et «normalisateur» de la connaissance scientifique la plus avancée. En s'engageant dans l'histoire des sciences, la philosophie peut «découvrir » les lignes de force de ce qu'elle considère comme le sommet de la connaissance et l'expression la plus haute de la pensée humaine. Certes, l'accord est difficile à réaliser et les différences ne tardent pas à se manifester, malgré l'utilisation d'une seule et même histoire, et donc bien que le passage par l'histoire des sciences soit devenu une nécessité pour tous ceux qui veulent sauvegarder la solidité et la valeur de la pensée rationnelle: les conclusions de Brunschvicg sont très différentes de celles de Meyerson ou de celles de Le Roy (un peu moins de celles de Rey). Tous, cependant, concordent sur la stratégie à suivre : l'histoire des sciences utilisée par les philosophes doit conduire le raisonnement à démontrer l'inébranlable force de la connaissance rationnelle. On veut donc défendre les droits de la raison humaine par ce que j'appelle un « humanisme de la raison », humanisme qui fait en partie descendre la raison elle-même de son piédestal et la fait « vivre » dans « ce monde-ci », un monde marqué par le temps qui coule, donc un monde historique. L'épistémologie n'est possible qu'à cette condition.

Pour aboutir à ce résultat — qui est un but établi presque explicitement $a$ priori - chacun suit son chemin et doit s'ouvrir toujours davantage à la possibilité d'assouplir l'architecture de la Raison : Brunschvicg par le rôle qu'il reconnaît à la négation et à la résistance du réel, Meyerson par l'introduction de ce qu'il appelle des irrationnels, Rey par la relativisation de la connaissance elle-même, etc. (mais ce point nous conduit à une problématique trop vaste pour être développée ici $^{26}$ ).

24. HeIDEgGer, 1959, chap. I.

25. BACHELARD, 1938, p. 22 (souligné par moi).

26. J'ai consacré une partie de Castelli Gattinara, 1998b, à cette question. 
Rey, Brunschvicg et Meyerson mènent en ce sens le même combat, repris ensuite par Koyré (qui partageait plutôt les idées de Meyerson) et Bachelard (qui penchait au contraire du côté de Rey et de Brunschvicg). Dans les textes publiés à partir des années dix, et notamment dès 1920, le rationalisme se manifeste en France comme un «rationalisme expérimental » dialectiquement ouvert, ce qui implique nécessairement l'insertion de l'histoire. Cela est imposé par la dialectique entre raison et expérience, et le schéma en est sommairement le suivant : les théories portent à la formulation d'hypothèses qui suscitent une certaine expérience en organisant et en structurant un certain ordre de réalité et ladite expérience se heurte à une réalité plus complexe et non-maîtrisable capable, en certains cas, de ne pas confirmer les hypothèses en amont — résistance, irrationnels, etc.; la réalité ainsi organisée et construite n'est pas inerte, mais réagit sur l'expérience qui ne s'y adapte que de façon approximative, et par là oblige à une révision des hypothèses, voire des théories, qui exigent ainsi en retour des expériences différentes, modifiant la constitution de la réalité, qui se rabat à nouveau sur les hypothèses, et ainsi de suite.

Or, dans cette conception, l'histoire des sciences joue un double rôle : d'abord, celui d'expérience cruciale, qui fait éclater le problème de l'évolution, du changement des théories et de la configuration du réel, en mettant en cause la rigidité architecturale de la raison; mais, aussi, celui d'hypothèse directrice, car si le mouvement de la raison est conçu comme une dialectique, ce mouvement ne tourne pas en rond mais se caractérise par un changement intrinsèquement nécessaire et progressif - continu ou discontinu. D'où, par conséquent, la connotation nécessairement progressive de la connaissance, puisque le mouvement dialectique fonctionne afin d'optimiser sans arrêt le rapport entre la théorie et l'expérience. L'histoire des sciences, malgré la « fragilité » que lui reproche Poincaré, témoigne de ce rapport dialectique; mais en même temps, elle est instituée par cette dialectique même. Sans l'histoire, la dialectique de la raison expérimentale n'a pas de sens. Mais sans sa dialectique intrinsèque, la raison ne donne pas de possibilité historique aux connaissances humaines.

La raison scientifique, en s'autolégitimant philosophiquement en tant qu'historique, constitue une sorte de « réponse » française à la crise de la raison, à la crise des fondements, à la crise de la civilisation. Elle répond non pas à l'exigence duhémienne de «sauver les phénomènes », mais à celle de «sauver la raison ». Une fois historicisée, la raison peut faire des crises et des bouleversements le sens de son propre mouvement, car toute crise représente le moment positif d'une évolution qui aboutira à une nouvelle configuration des connaissances. En s'humanisant, elle devient historique. 
Le passage par l'histoire que le congrès de Philosophie de 1900 avait ébauché devient donc toujours plus indispensable, bien qu'il se fasse sous l'hégémonie du savoir philosophique. Le but principal d'un penseur qui s'occupe des sciences est de réussir à jouer un rôle moralisateur et normalisateur. L'épistémologie s'est en effet constituée en France aux dépens de l'histoire des sciences. Et la présence d'un grand nombre de philosophes en position dominante dans les institutions et les revues qui s'occupent d'histoire des sciences dans l'entre-deux-guerres nous permet de comprendre l'une des conséquences les plus radicales de cette situation. Il faut évoquer, une fois encore, les propos de Bachelard, si explicites qu'ils se passent de commentaire :

«À côté de l'histoire de ce qui fut, alentie et hésitante, on doit écrire une histoire de ce qui aurait dû être, rapide et péremptoire. Cette histoire normalisée, elle est à peine inexacte. Elle est fausse socialement, dans la poussée effective de la science populaire qui réalise [...] toutes les erreurs. Elle est vraie par la lignée des génies, dans les douces sollicitations de la vérité objective. C'est cette ligne légère qui dessine le destin véritable de la pensée humaine ${ }^{27}$. »

Voilà comment la légèreté du philosophe peut s'exercer lourdement et sans appel. Le «destin véritable » de la pensée humaine - ce souci qui avait commencé à inquiéter sérieusement les philosophes au tournant du siècle et les avait poussés à se réunir pour y faire face - , ce destin trouve dans l'histoire un instrument formidable pour s'autolégitimer. Mais l'histoire reste une arme à double tranchant, qui doit être maitrisée par le métadiscours de la philosophie. Car seule la philosophie, parmi toutes les autres formes du savoir, est apte à donner un sens aux évolutions aveugles de l'histoire.

Face aux nouveaux problèmes posés par le $\mathrm{xx}^{\mathrm{e}}$ siècle et au retard accumulé à l'égard des nouvelles découvertes et des théories scientifiques, les philosophes français de la première moitié du siècle tentent donc un coup de force et le réussissent. Ils s'ouvrent stratégiquement à l'histoire à l'histoire des sciences - afin de récupérer et de maintenir un rôle de guide par rapport à toutes les sciences. Ils inaugurent ainsi une tradition épistémologique qui allait exister de 1900 jusqu'aux années soixante, et qui était soutenue par une conception assouplie et dynamique de la raison rendue possible par l'ouverture de la philosophie des sciences à leur histoire.

Mais le passage par l'histoire comporte aussi des « risques » que les philosophes, par exemple Bachelard, ont bien envisagé; d'où l'aspect péremptoire et hégémonique des propos de Bachelard sur la valeur d'une histoire

27. BaChelard, 1938, p. 251. 
épistémologique des sciences, d'une histoire «normalisée ». En effet, le discontinuisme, encore inconcevable dans les années vingt mais dominant à partir des années cinquante, passe par l'histoire des sciences. Avec le discontinuisme, une brèche s'ouvre dans la forteresse du dogmatisme philosophique et épistémologique. C'est Bachelard lui-même qui l'ouvrit, mais sans crainte, car elle était intégrée à son épistémologie. Cependant, par cette brèche discontinuiste, c'est le caractère différentiel de l'histoire qui s'impose à l'attention des chercheurs, la complexité de ses rapports et la stratification du réel (matériel, idéal, social, économique, historique, etc.), dont l'histoire ne peut s'abstraire qu'au prix d'une violence toute philosophique. En même temps, d'autres histoires des sciences étaient en train d'être écrites, toujours sous l'empire d'une conception philosophique, mais très documentées, comme celle de Koyré. Les sciences humaines, en outre, réclamaient de plus en plus leurs droits. Un autre tournant critique s'annonce donc à partir des années soixante-dix, et une autre tradition commence, où la place dominante de la philosophie sera définitivement mise en cause, et peut-être complètement éliminée. L'histoire des sciences trouvera d'autres alliés chez les sociologues ou chez les anthropologues, peut-être moins exigeants que les philosophes. Mais il s'agit alors de notre propre époque, et il est peut-être encore trop tôt pour en proposer l'analyse.

Enrico Castelli Gattinara (juin 2000). 


\section{LISTE DES RÉFÉRENCES}

BACHELARD (Gaston), 1938, La Formation de l'esprit scientifique, Paris, Vrin.

Bachelard (G.), 1972, «L'actualité de l'histoire des sciences », in ID., L'Engagement rationaliste, Paris, Presses universitaires de France.

Boutroux (Émile), 1900, «Allocution » introduisant le congrès, « Premier congrès international de Philosophie », Paris, $1^{\mathrm{er}}-6$ août 1900, interventions et résumés, Revue de métaphysique et de morale, t. VIII, p. 503-511.

BRunEtiÈRE (Ferdinand), 1895, "Après une visite au Vatican », Revue des deux mondes, janv. 1895 , p. 97-118.

Castelli Gattinara (Enrico), 1997, «L'idée de la synthèse. Henri Berr et les crises du savoir dans la première moitié du $\mathrm{xx}^{\mathrm{e}}$ siècle », in Henri Berr et la culture du $\mathrm{XX}^{e}$ siècle. Histoire, science et philosophie, actes du colloque international, Paris, 24-26 oct. 1994, dir. Agnès BiARD, Dominique Bourel et Éric Brian, Paris, Albin Michel/Centre international de synthèse, 1997, p. 21-38.

Castelli Gattinara (E.), 1998a, «Épistémologie, histoire et histoire des sciences dans les années 1930,1 et 2 », Revue de synthèse, t. CXIX, $4^{\mathrm{e}}$ sér., 1 , janv.mars, p. 9-36 et p. 37-61.

Castelli Gattinara (E.), 1998b, Les Inquiétudes de la raison, Paris, École des hautes études en sciences sociales/Vrin.

Coumet (Ernest), 1981, «Paul Tannery. L'organisation de l'enseignement de l'histoire des sciences », Revue de synthèse, t. CII, $3^{\mathrm{e}}$ sér., 101-102, janv.-juin, p. 87-123.

HeIDEGGER (Martin), 1959, Qu'appelle-t-on penser?, trad. franç. par Aloys BECKER et Gérard Granel, Paris, Presses universitaires de France.

LaLANDE (André), 1900, «Le congrès international de Philosophie », Revue philosophique, t. L, 32, p. 481-508.

Meyerson (Émile), 1908, Identité et réalité, Paris, Vrin.

REY (Abel), 1907, «Le mécanisme et l'énergétique », Revue philosophique, t. LXIV, 46, p. 495-517.

TANNERY (Paul), 1900, «Morale », discussion finale de la dernière séance du premier congrès international de Philosophie, Revue de métaphysique et de morale, t. VIII, p. 694-698.

VAléry (Paul), 1973, Cahiers, Paris, Gallimard, vol. I et II.

Wilbors (Joseph), 1900, «Argument en faveur de la liberté humaine tiré du déterminisme physique », "Premier congrès international de Philosophie », Paris, $1^{\mathrm{er}}-6$ août 1900, interventions et résumés, Revue de métaphysique et de morale, t. VIII, p. 540-542. 